\title{
Detection of bone marrow invasion by neuroblastoma is improved by sampling at two sites with both aspirates and trephine biopsies
}

\author{
IM FRANKLIN,* J PRITCHARD \\ From the Department of Haematology and Oncology, The Hospital for Sick Children, Great Ormond Street, \\ London WC1N $3 J H$
}

SUMMARY Two hundred and eight serial bone marrow samples from 49 consecutively diagnosed children with neuroblastoma were studied retrospectively for evidence of tumour invasion. Bone marrow involvement was found in 24 patients at diagnosis and in four more at a later stage in their disease. Trephine biopsies were more effective than aspirates for tumour detection in $20 \%$ of the 154 paired aspirate/trephine procedures, whilst the reverse was the case in $7 \%$. Imprints of trephines gave no additional information. Bilateral sampling (aspirates and trephines) improved the tumour detection rate by $10 \%$ over that attained by sampling a single site. There was some correlation between specific appearances in aspirate and in trephine samples, for example the hypocellular smear and the trephine biopsy showing much stromal reaction to tumour infiltration.

Bilateral iliac crest bone marrow aspirates and trephine biopsies are indicated in children with neuroblastoma, both for initial staging and for monitoring of progress.

Neuroblastoma is a relatively common childhood tumour and patients with advanced disease often have bone marrow involvement. ${ }^{1}$ Staging criteria, ${ }^{2}$ including marrow sampling, ${ }^{3}$ have assisted in defining poor prognosis patients. The recent use of autologous bone marrow "rescue", following high dose chemotherapy, ${ }^{4}$ has provided an additional important reason for accurate assessment of bone marrow status of such patients. In adults, it is well recognised that invasion of marrow by solid tumours may have a focal distribution ${ }^{56}$ that may be missed by examining only aspirated material or by sampling a single site: trephine biopsy and bilateral sampling have therefore been suggested to improve the detection rate. $^{5}$ Trephine biopsies have also been recommended in children ${ }^{7}$ but, to date, there has been no large-scale study of their value in patients with neuroblastoma.

Since 1977 we have performed bilateral posterior iliac crest bone marrow aspirates and trephine biopsies on children for staging of neuroblastoma, for monitoring response to chemotherapy and for assessing suitability for bone marrow harvesting. In this

\footnotetext{
*Present address: Department of Haematology, The Queen
} Elizabeth Hospital, Edgbaston, Birmingham B15 2TH.

Accepted for publication 14 July 1983 paper we analyse the results of these investigations in order (a) to assess the practicability of trephine biopsy in young children (b) to compare the efficacy of aspirate and trephine biopsy for detection of metastatic disease (c) to assess the role of simultaneous marrow sampling at two sites and (d) to identify patterns of marrow involvement by neuroblastoma.

\section{Patients and methods}

Bone marrow aspirates and trephine biopsies from 49 patients with neuroblastoma referred to the Hospital for Sick Children between 1977 and 1981 were studied retrospectively for evidence of infiltration by tumour.

Diagnosis was either by histopathological examination of primary or secondary tumour or by a combination of clinical, radiological and biochemical findings. ${ }^{8}$ There were 28 boys and 21 girls, aged between 1 month and 12 yr (median 33 months). Apart from bone marrow examination, staging was by chest radiography, abdominal ultrasound with or without CT scanning, bone scan and skeletal survey; the distribution of stages was typical for any moderate or large series of children with neuroblastoma (Table 1). Of the 208 procedures, 86 were per- 
Table 1 Patients and procedures

\begin{tabular}{lclc}
\hline Stage & $\begin{array}{l}\text { No of } \\
\text { patients }\end{array}$ & $\begin{array}{l}\text { Marrow involved } \\
\text { at diagnosis }\end{array}$ & $\begin{array}{c}\text { Total No of } \\
\text { procedures }\end{array}$ \\
\hline I & 4 & - & 7 \\
II & 6 & - & 11 \\
III & 5 & - & 19 \\
IV & 34 & 24 & 171 \\
Total & 49 & 24 & 208 \\
\hline
\end{tabular}

*Four additional patients (1 previously stage III; 3 stage IV) had marrow invasion detected during follow-up.

formed at diagnosis and the remaining 122 at follow-up.

Bone marrow involvement was assessed by examination of spread films of aspirated material, stained by May-Grünwald-Giemsa. All available slides (median 3-4) were examined thoroughly for evidence of gross disease or for small clumps of tumour cells. In the absence of clumps, a differential count of at least 500 nucleated cells was performed before a specimen was declared free of tumour. Sections of formalin-fixed trephine biopsies, obtained from the posterior iliac crest using the Jamshidi needle under ketamine anaesthesia, were decalcified in Zenker's medium, stained with haematoxylin and eosin and then examined systematically at low and high magnification; Giemsa-stained sections and others stained for reticulin were also examined. Imprint ("touch") preparations of bone marrow trephine biopsies were examined if available.

\section{Results}

Of the 208 aspirates and/or trephine biopsies examined, 33 consisted only of an aspirate (29) or a trephine biopsy (4). A further 21 marrow aspirates had a corresponding trephine biopsy that was inadequate in that it was either crushed (4), only consisted of cartilage (7) or was a tiny bone chip with no marrow spaces (10). The median age of the children at the time of an inadequate or unavailable trephine biopsy was 33 months, the same as the whole group. However, for children younger than the median age, there were 23 failures out of 64 attempts (success rate of $64 \%$ ), compared with 25 failures in 144 attempts ( $82 \%$ successful) on older children.

\section{COMPARISON OF ASPIRATE AND TREPHINE BIOPSIES}

One hundred and fifty-four pairs of aspirate and trephine biopsies were examined, of which 122 represented 61 simultaneous bilateral pairs. Trephine biopsy detected tumour more often than aspirates, but in 10 instances only the aspirate was positive (Table 2).
Table 2 No of paired aspirates and trephine biopsies demonstrating tumour invasion

\begin{tabular}{ll}
\hline Aspirate and trephine biopsy positive & 31 \\
Trephine biopsy positive & 31 \\
Aspirate positive & 10 \\
\hline These represent $47 \%$ of the total aspirate and trephine biopsy \\
pairs.
\end{tabular}

BILATERAL SAMPLING

Of the 61 pairs of simultaneous bilateral aspirate and trephine samples, either the aspirate or trephine biopsy was positive on one side only in 13 pairs $(21 \%)$. Both sides were positive, and both negative, in 24 instances each.

\section{IMPRINT (TOUCH) PREPARATIONS FROM \\ TREPHINE BIOPSIES}

Ninety-four imprint preparations were examined but 26 were technically unsatisfactory, consisting only of amorphous debris. Only one of the remaining 68 samples yielded information not obtained from aspirate or trephine: in this instance, the aspirate was negative but a single clump of tumour cells, detached from a crushed (and therefore inevaluable) trephine sample, was identified on the imprint preparation. There was one other case where the imprint was positive and the aspirates negative but, in this instance, the corresponding trephine biopsy was also positive for tumour.

\section{PATTERNS OF TUMOUR INVASION}

Certain microscopic features of involved aspirates and trephine biopsies were noted. Unsatisfactory, dilute aspirates with many disrupted cells but no obvious tumour were associated in 12 of 16 such samples with a trephine biopsy that showed extensive involvement with neuroblastoma and a marked stromal reaction (Fig.). Only twice was a dilute aspirate associated with a corresponding normal trephine sample, and the remaining two cases showed extensive invasion with neuroblastoma without stromal reaction.

The presence of classical neuroblastoma "rosettes" was uncommon, and was seen only twice in aspirated material. In those cases with focal involvement, tumour was surrounded by normal marrow and no evidence of any lymphoid reaction was seen. ${ }^{9}$

\section{Discussion}

The 208 available bone marrow aspirates were paired with 154 trephine samples that demonstrated some marrow tissue, representing a $74 \%$ success rate for trephine biopsy. Cozzutto et al ${ }^{7}$ obtained an 

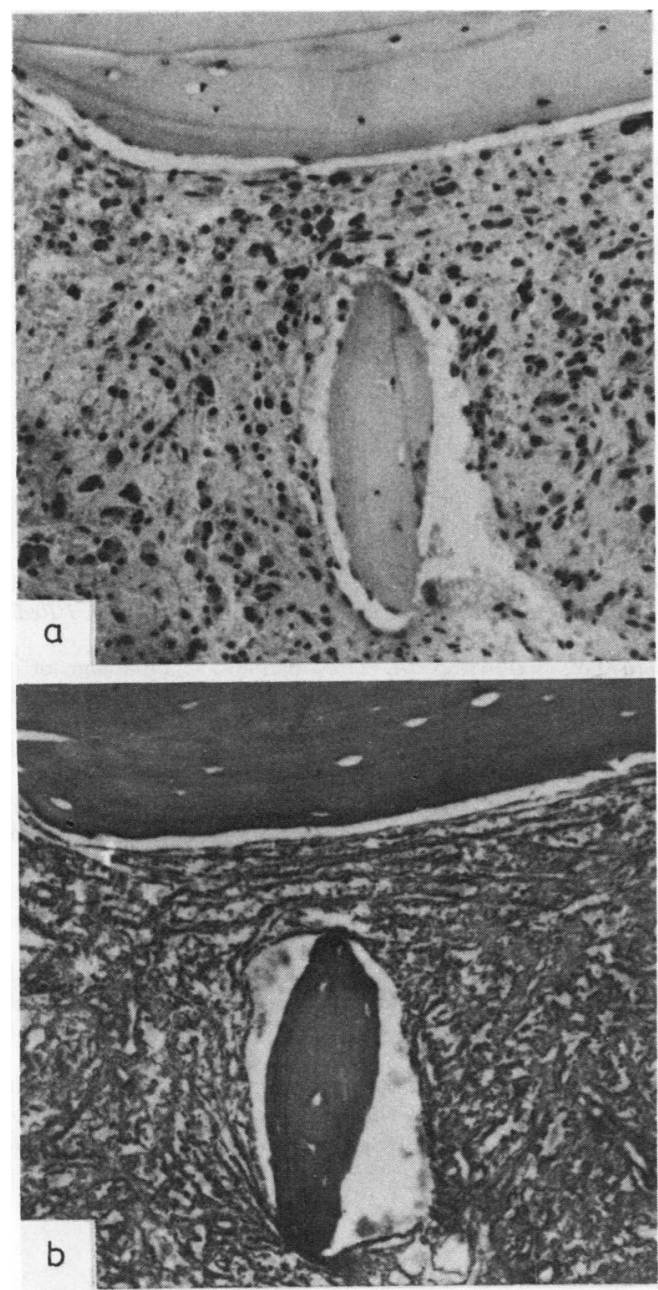

Section of trephine bone biopsy showing replacement of bone marrow with neuroblastoma associated with a marked stromal reaction (a) stained with haematoxylin and eosin which is associated with prominent reticulin deposition; (b) stained for reticulin with silver impregnation method.

adequate sample in over $80 \%$ of attempts at trephine biopsy in children under $15 \mathrm{yr}$ of age, which is comparable with that achieved in this study for children under $12 \mathrm{yr}$. Those cases where the trephine was either unavailable or inadequate had the same median age, 33 months, as the whole group, but the success rate in children aged less than this was $64 \%$ whereas for older children it was $82 \%$. Of the four trephines with no corresponding aspirate, three had fibrotic marrow involvement with tumour.

Bone marrow was found to be involved at diag- nosis in 24 of 49 children. In most of these, there was evidence of metastatic spread at other sites. A similar incidence of marrow involvement at diagnosis was found by Cozzutto et al (7 of 12 trephines). ${ }^{7}$ Finkelstein et al, ${ }^{3}$ using aspirates only, detected invasion of marrow in 28 of 40 cases, but it is not known whether these were a selected group.

Four children in whom marrow invasion was not detected at presentation subsequently had positive aspirates or biopsies, demonstrating the importance of marrow sampling in assessing the progress of children with neuroblastoma.

Only the trephine biopsy was positive for tumour in $31(20 \%)$ of the 154 aspirates and trephine sample pairs but the importance of performing both procedures is confirmed by the 10 cases $(7 \%)$ when the aspirate provided the only evidence of tumour. The overall superiority of trephine biopsy in neuroblastoma accords with experience in adult malignancies except for diffuse lymphomas and leukaemias. ${ }^{111}$ These two tumours do not produce a stromal reaction nor tend to form focal patterns of infiltration, both of which are features of neuroblastoma and contribute to the likelihood of sampling errors.

At diagnosis, 6 of 60 aspirate and trephine biopsy pairs showed a discrepancy with only the trephine sample being involved with tumour. However, 25 of 94 follow-up procedures had only the trephine sample positive, more than twice the proportion at diagnosis. A marked stromal reaction in the trephine biopsy was also more common on follow-up sampling, with 19 examples against 9 at diagnosis. Increased stromal reaction to neuroblastoma following chemotherapy might explain these findings.

The simultaneous bilateral aspirates and trephines from the posterior iliac crests confirm the value of sampling at these two sites, since on $21 \%$ of occasions only one side was positive. In clinical practice this is only a $10 \%$ improvement because half would be discovered on the "first" side, but this appears a worthwhile gain and does not discomfit the anaesthetised child. The role of marrow aspirates at multiple sites has been studied by Hartmann ( $O$ Hartmann, personal communication, 1983) and is being assessed at this hospital.

The presence of very small aggregates of neuroblastoma in otherwise normal marrow aspirates makes an exhaustive search of all available films mandatory. Diffuse involvement of marrow with neuroblastoma is also difficult to diagnose if abnormal cells are less than $5 \%$, since neuroblasts may appear similar to normal lymphoid cells. ${ }^{12}$ The differential count may be difficult to interpret therefore, but is recommended because diffuse involvement with a larger proportion of cells might be over- 
looked if films are scanned for clumps only. Tumour cell "rosettes", usually considered to be typical of neuroblastoma in bone marrow, were seen in less than $2 \%$ of samples, and contribute to the diagnosis of marrow involvement with neuroblastoma most infrequently. An unsatisfactory dilute or "blood" tap in a suspected case of neuroblastoma represents a particular indication for trephine biopsy and is presumptive evidence of marrow invasion.

The diagnosis of marrow infiltration with neuroblastoma remains imprecise, in particular because of its tendency for focal invasion. Monoclonal antibodies directed against neuroblasts increase the sensitivity and objectivity of the diagnosis, ${ }^{13}$ but the problem of "sampling error" remains. Radioisotope scans using such monoclonal antibodies as carriers might overcome this problem. ${ }^{14}$

We conclude that combined bone marrow aspiration and trephine biopsy are relatively easy to perform in young children, and that they both contribute to the staging and re-assessment of children with neuroblastoma. It is worth performing both procedures at two sites, but the value of sampling more marrow sites than this is at present not known. The focal nature of metastatic spread means that (a) the identification of small numbers of single tumour cells and (b) sampling errors will always present problems and alternative methods for even more efficient detection of marrow involvement, particularly radioimmunolocalisation techniques, should be urgently investigated.

We wish to thank Dr JR Pincott and Mr G Anderson of the Department of Histopathology for providing the trephine sections. JP received financial support from the Leukaemia Research Fund and Imperial Cancer Research Fund.

\section{References}

' Kinnear Wilson MA, Draper GJ. Neuroblastoma, its natural history and prognosis: a study of 487 cases. $\mathrm{Br}$ Med J 1974;iii:301-7.
${ }^{2}$ Evans AE, D' Angio GF, Randolph F. A proposed staging for children with neuroblastoma. Cancer 1970;27:374-8.

${ }^{3}$ Finkelstein JZ, Ekert $\mathbf{H}$, Isaacs $\mathrm{H}$, Higgins $\mathbf{G}$. Bone marrow metastases in children with solid tumors. Am J Dis Child 1970;119:49-52.

4 Pritchard J, McElwain TJ, Graham-Pole J. High-dose melphalan with autologous marrow for treatment of advanced neuroblastoma. Br J Cancer 1982;45:86-94.

${ }^{5}$ Brunning RD, Bloomfield CD, McKenna RW, Peterson L. Bilateral trephine bone marrow biopsies in lymphoma and other neoplastic diseases. Ann Intern Med 1975;82:365-6.

- Contreras E, Lawrence DE, Lee RE. Value of the bone marrow biopsy in the diagnosis of metastatic carcinoma. Cancer 1972;29:778-85.

' Cozzutto C, De Bernardi B, Comelli A, Guarino M. Bone marrow biopsy in children: a study of 111 patients. Med Pediatr Oncol 1979;6:57-64.

- Pritchard J, Kemshead JT. Neuroblastoma-recent developments in diagnosis, staging and management. Proc $R$ Soc Radiol 1983: (in press).

' Martin RF, Beckwith JB. Lymphoid infiltrates in neuroblastomas: their occurrence and prognostic significance. $J$ Pediatr Surg 1968;3:161-4.

${ }^{10}$ Dee JW, Valdivieso M, Drewinko B. Comparison of the efficacies of closed trephine needle biopsy, aspirated paraffinembedded clot section, and smear preparation in the diagnosis of bone marow involvement by lymphoma. Am J Clin Pathol 1976;65:183-94.

" Grann V, Pool JL, Mayer K. Comparative study of bone marrow aspiration and biopsy in patients with neoplastic disease. Cancer 1966;19:1898-1900.

${ }^{12}$ Evans AE, Hummeler $\mathrm{K}$. The significance of primitive cells in marrow aspirates of children with neuroblastoma. Cancen 1973;32:906-12.

${ }^{13}$ Kemshead JT, Goldman A, Fritschy J, Malpas JS, Pritchard Use of panels of monoclonal antibodies in the differentia diagnosis of neuroblastoma and lymphoblastic disorders. Lan $?$ cet 1983;i:12-14.

14 Goldman A, Pritchard J, Kemshead JT. Selective localization of radiolabelled monoclonal antibodies to human neuroblastoma xenografts in a nude mouse model. Proc Am Soc Clin Oncol 1983; (in press).

Requests for reprints to: Dr IM Franklin, Department of Haematology, The Queen Elizabeth Hospital, Edgbaston, Birmingham B15 2TH, England. 